\title{
SEPSIS SYNDROME
}

Sepsis syndrome is defined as infection associated with the Systemic Inflammatory Response Syndrome (SIRS). Sepsis has various etiologies and clinical presentations. It accounts for substantial morbidity and mortality. The Healthcare Cost and Utilization Project (HCUP) estimated 300,000 discharges for sepsis syndrome in 2002, with an in-hospital mortality of $18.6 \%$. The mean length-of-stay was 7.3 days with approximately $\$ 26,000$ in charges per patient. Sepsis requires expeditious diagnosis and standardized treatment plans to favorably impact patient morbidity and mortality. Hospitalists play a key role in the early identification of patients with sepsis, and practice aggressive evidence based evaluation and interventions. Hospitalists lead their institutions to implement early diagnostic strategies, initiate evidence based medical therapies, and incorporate multidisciplinary approaches to the care of patients with sepsis.

\section{KNOWLEDGE}

Hospitalists should be able to:

- Define and differentiate bacteremia and the clinical spectrum of SIRS, sepsis, severe sepsis, and septic shock.

- Describe the symptoms and signs of SIRS, sepsis, severe sepsis, and septic shock.

- Describe the inflammatory cascade that leads to SIRS and sepsis.

- Distinguish infectious causes of SIRS from other etiologies.

- Distinguish septic shock from other causes of shock.

- Describe the indicated tests required to evaluate sepsis.

- Identify patient groups with increased risk for the development of sepsis, increased morbidity or mortality, or uncommon etiologic organisms.

- Discuss the evidence based diagnostic choices available in the evaluation of sepsis.

- Describe the indications, contraindications and side effects of therapeutic agents including fluids, vasopressors, antibiotics, steroids, activated protein $\mathrm{C}$, and blood products in the treatment of sepsis.

- Explain indications, contraindications and mechanisms of action of pharmacologic agents used to treat sepsis syndrome.

- Describe the indications for and limitations of central venous access and its value for hemodynamic monitoring and administration of vasoactive agents.

- Describe the role of established scoring systems to estimate the severity of sepsis.

- Explain patient characteristics that on admission portend poor prognosis.

- Explain goals for hospital discharge, including specific measures of clinical stability for safe care transition.

\section{SKILLS}

Hospitalists should be able to:

- Utilize all available information, including medical records and history provided by patient and care givers, to identify factors that contribute to the development of sepsis.

- Perform a rapid and targeted physical examination to identify potential sources of sepsis.

- Recognize the value and limitations of the history and physical examination in determining the cause of sepsis.

- Order indicated diagnostic testing to identify the source of sepsis and determine severity of organ dysfunction.

- Rapidly identify patients with septic shock and aggressively treat in parallel with transfer to a critical care setting.

- Assess cardiopulmonary stability and implement aggressive fluid resuscitation, airway maintenance and circulatory support.

- Initiate empiric antimicrobial therapy based on the suspected etiologic source of infection.

- Assess the need for central venous access and monitoring; when needed, coordinate or establish central venous access.

- Determine or coordinate appropriate nutritional and metabolic interventions.

- Support organ function and correct metabolic derangements when indicated.

- Implement measures to ensure strict glycemic control.

- Adopt measures to prevent complications, which may include aspiration precautions, stress ulcer and VTE prophylaxis, and decubitus ulcer prevention.

- Measure and interpret indicated hemodynamic monitoring parameters. 


\section{ATTITUDES}

Hospitalists should be able to:

- Communicate with patients and families to explain the history and prognosis of sepsis and indicators of functional improvement or decline.

- Communicate with patients and families to explain goals of care plan, including clinical stability criteria, discharge instructions and management after release from hospital.

- Communicate with patients and families to explain tests and procedures and their indications, and to obtain informed consent.

- Recognize the indications for specialty consultations, which may include critical care medicine.

- Employ an early and multidisciplinary approach, which may include respiratory therapy, nursing, pharmacy, nutrition, rehabilitation and social services, that begins at admission and continues through all care transitions.

- Establish and maintain an open dialogue with patients and families regarding care goals and limitations, including palliative care and end-of-life wishes.

- Address resuscitation status early during hospital stay, and discuss and implement end of life decisions by patient or family when indicated or desired.

- Ensure good communication with patients and receiving physicians during care transitions.

- Utilize evidence based recommendations to guide diagnosis, monitoring and treatment of sepsis.

\section{SYSTEM ORGANIZATION AND IMPROVEMENT}

To improve efficiency and quality within their organizations, Hospitalists should:

- Lead, coordinate or participate in the development and promotion of guidelines and/or pathways that facilitate efficient and timely evaluation and treatment of patients with sepsis.

- Implement systems to ensure hospital-wide adherence to national standards, and document those measures as specified by recognized organizations.

- Lead, coordinate or participate in multidisciplinary initiatives to promote patient safety and optimize resource utilization.

- Lead, coordinate or participate in intra- and inter-institutional efforts to develop protocols for the rapid identification and transfer of patients with sepsis to appropriate facilities.

- Lead, coordinate or participate in multidisciplinary teams, which may include nutrition, pharmacy, rehabilitation, social services and respiratory therapy, early in the hospital course to improve patient function and outcomes.

- Integrate outcomes research, institution-specific laboratory policies, and hospital formulary to create indicated and cost-effective diagnostic and management strategies for patients with sepsis. 